\title{
In-Situ EDS Characterization of TEM Lamellae Created by Xe Plasma FIB
}

\author{
Tomáš Hrnčîrir ${ }^{1}$, Christian Lang ${ }^{2}$, Jozef Vincenc Oboňa ${ }^{1}$ and Tomáš Barták ${ }^{3}$ \\ 1. TESCAN, Brno, Czech Republic \\ 2. Oxford Instruments Nanoanalysis, High Wycombe, UK \\ ${ }^{3 .}$ Oxford Instruments, Prague, Czech Republic
}

As a result of the rapidly progressing miniaturization in microelectronics, the critical dimensions of semiconductor devices are now so small that only TEM analysis can give conclusive answers with regard to fault isolation and failure analysis. In order to investigate structures on the nanometer and subnanometer scale, ultrathin TEM lamellae have to be prepared that contain the fault in question. As site specific preparation is crucial and samples are precious, FIB-SEM has become the standard tool for the preparation of TEM lamellae for failure analysis. However, with increasing integration of devices and three dimensional packaging starting to be common now, it is becoming more difficult to rapidly locate the position of a fault with sufficient accuracy to prepare a TEM lamella using a conventional $\mathrm{Ga}^{+}$FIB in a reasonable time frame. The milling rates achievable in most $\mathrm{Ga}^{+}$FIBs restrict the maximum lamella dimensions in both width and height to tens of microns. This necessitates time consuming additional investigations to pinpoint the location of a defect before putting the sample into the FIB-SEM. Novel plasma FIB-SEMs employs $\mathrm{Xe}^{+}$beam which enables milling rates more than 50 times faster than conventional $\mathrm{Ga}^{+}$FIBs [1]. Therefore lamellae can be prepared rapidly with dimensions of hundreds of microns [2], saving time in isolating defects.

While a large lamella opens up the possibility to speed up failure analysis, the lamella quality is nevertheless as critical as ever in order to obtain good quality TEM images. Preparation artifacts need to be understood and eliminated as far as possible. Here we investigate lamellae prepared in TESCAN FERA3 FIB-SEM. All the process steps were done at $30 \mathrm{kV}$ acceleration voltage starting with rough milling at $1 \mu \mathrm{A}$ beam current and final polish at $1 \mathrm{nA}$. In order to accurately characterize the lamella thickness and any implantation of ions during the preparation process, we use in-situ EDS analysis in combination with a special software to calculate the lamella thickness (AZtec LayerProbe). This method has been shown to give accurate results for $\mathrm{Ga}^{+}$prepared lamellae of a range of materials [3]. The LayerProbe software refines a starting model of the sample structure against the EDS spectra to calculate the film thickness and composition of the layers. The starting model comprises the layer sequence in the sample and a substrate material. As the TEM lamella is a free-standing layer the substrate is defined as comprising an element that is not contained in the lamella and only weakly scatters electrons such as Beryllium. The first layer is defined as the material comprising the lamella and the element implanted by the ion beam. The thickness of that layer corresponds to the lamella thickness.

Figure 1 shows an electron image with an overlayed X-ray map of the whole lamella. There are three regions of different thickness, varying from around 2 micron down to 0.2 micron (cf. figure 2). The EDS map clearly indicates a Pt rich layer on top of the lamella which was deposited to protect the lamella during the Xe milling process. A map of the Xe-L line indicates that this top layer also contains significant amounts of Xe ions whereas the rest of the lamella shows very little Xe implantation. This difference in the Xe content between the Pt layer and the rest of the lamella can be explained by the fact that to deposit $\mathrm{Pt},\left(\mathrm{CH}_{3}\right)_{3}\left(\mathrm{CH}_{3} \mathrm{C}_{5} \mathrm{H}_{4}\right) \mathrm{Pt}$ gas was injected and decomposed by a vertically incident Xe beam. 
However, during the preparation of the lamella, the Xe beam was nearly parallel to the lamella and Xe ions were deflected off the sides rather than being implanted.

To quantify the amount of Xe in the Pt rich top layer and the Si below two X-ray spectra were taken and the Xe composition was calculated by assuming a homogeneous distribution of Xe throughout the material (figure 3). The results show that the Pt rich layer contains around 3 at\% Xe and 79 at\% C. This can be compared to previous studies on Ga ion beam induced deposition [4]. The Pt content is similar whether Xe or Ga ions are used, the carbon content is higher when depositing with Xe but the implanted ion content lower. The levels of Xe implanted in the lamella itself are below the detection limit of EDS technique (figure 3). Xe seems not to be as readily implanted as Ga which may be due to differences in the atomic radius of the ions. Further studies are in progress to corroborate these results in the TEM.

References:

[1] T. Hrnčír et al., 38th ISTFA Conference Proceedings (2012), p. 26.

[2] A. Delobbe et al., Microscopy and Microanalysis 20 (2014), p. 298.

[3] C. Lang et al., Microelectronics Reliability 54 (2014), p. 1790.

[4] C. Lang et al., Microscopy and Microanalysis 18 (2012), p. 620.

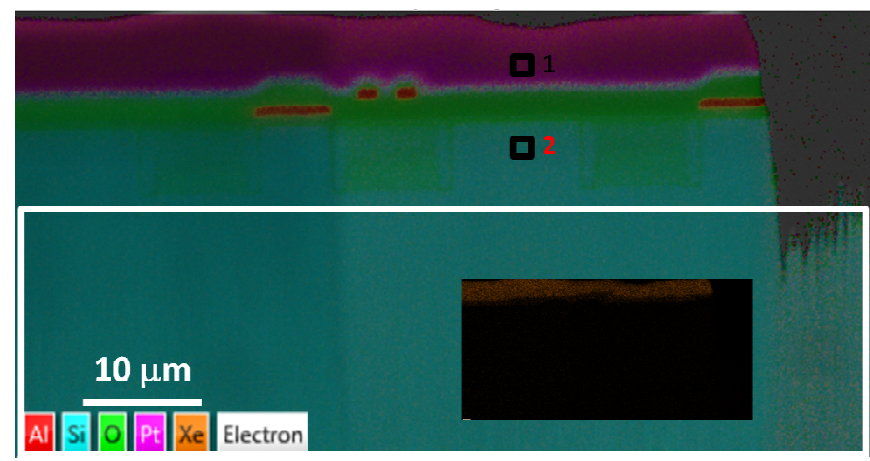

Figure 1 shows an electron image and X-ray map of the lamella prepared in Xe plasma FIB. The inset shows the Xe map. The large rectangle indicates the area over which the thickness was mapped (figure 2). The small squares indicate area from which spectra were taken for the Xe implantation measurements (figure 3).

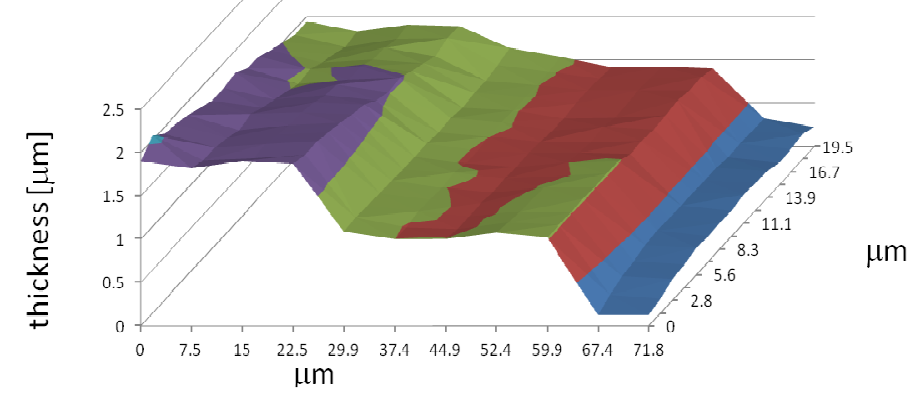

Figure 2 shows a thickness map of part of the lamella as indicated in figure 1.

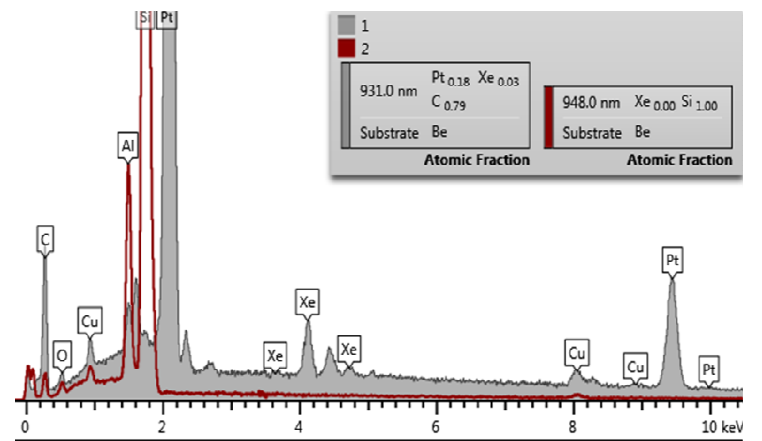

Figure 3 shows two X-ray spectra (1) and (2). (1) is from the Pt rich top layer and (2) from an area just below containing only $\mathrm{Si}$. The $\mathrm{Cu}$ and $\mathrm{Al}$ peaks are stray X-rays from the sample holder and grid post. 